\title{
Long non-coding RNA CASC9 promotes gefitinib resistance in NSCLC by epigenetic repression of DUSP1
}

\author{
Zhenyao Chen', Qinnan Chen', Zhixiang Cheng' ${ }^{1}$ Jingyao Gu', Wenyan Feng ${ }^{1}$, Tianyao Lei ${ }^{1}$, Jiali Huang ${ }^{1}$, Jiaze Pu², \\ Xin Chen ${ }^{1}$ and Zhaoxia Wang ${ }^{1}$
}

\begin{abstract}
Resistance to epidermal growth factor receptor tyrosine kinase inhibitors (EGFR-TKIs), such as gefitinib, has greatly affected clinical outcomes in non-small cell lung cancer (NSCLC) patients. The long noncoding RNAs (IncRNAs) are known to regulate tumorigenesis and cancer progression, but their contributions to NSCLC gefitinib resistance remain poorly understood. In this study, by analyzing the differentially expressed IncRNAs in gefitinib-resistant cells and gefitinib-sensitive cells in the National Institute of Health GEO dataset, we found that IncRNA CASC9 expression was upregulated, and this was also verified in resistant tissues. Gain and loss of function studies showed that CASC9 inhibition restored gefitinib sensitivity both in vitro and in vivo, whereas CASC9 overexpression promoted gefitinib resistance. Mechanistically, CASC9 repressed the tumor suppressor DUSP1 by recruiting histone methyltransferase $\mathrm{EZH} 2$, thereby increasing the resistance to gefitinib. Furthermore, ectopic expression of DUSP1 increased gefitinib sensitivity by inactivating the ERK pathway. Our results highlight the essential role of CASC9 in gefitinib resistance, suggesting that the CASC9/EZH2/DUSP1 axis might be a novel target for overcoming EGFR-TKI resistance in NSCLC.
\end{abstract}

\section{Introduction}

Non-small cell lung cancer (NSCLC) is one of the most common malignant tumors worldwide. Despite continuous developments and progress in therapeutic measures, such as surgery, radiotherapy, and drug therapy (including chemotherapy, targeted therapy, and immunotherapy), the prognosis of patients with advanced NSCLC is still poor ${ }^{1,2}$. The first generation of epidermal growth factor receptor tyrosine kinase inhibitors (EGFR$\mathrm{TKI})$, represented by gefitinib, could significantly prolong

\footnotetext{
Correspondence: Zhaoxia Wang (zhaoxiawang66@126.com) or Xin Chen (chen_xin4945@163.com)

${ }^{1}$ Cancer Medical Center, The Second Affiliated Hospital of Nanjing Medical University, Nanjing, Jiangsu, People's Republic of China

${ }^{2}$ Department of Oncology, The Fourth Affiliated Hospital of Nanjing Medical University, Nanjing, Jiangsu, People's Republic of China

These authors jointly supervised this work and should be regarded as joint first authors: Zhenyao Chen, Qinnan Chen, Zhixiang Cheng, Jingyao Gu Edited by I. Amelio
}

the median survival of patients with advanced lung cancer with EGFR-sensitive mutations and greatly improve their quality of life ${ }^{3}$. However, acquired resistance is inevitable with gefitinib, which leads to treatment failure ${ }^{4}$. Therefore, in-depth study of the mechanism of drug resistance to EGFR-TKIs, the search for genes related to drug resistance, and the exploration of ways to reverse drug resistance have recently become important topics in lung cancer treatment research.

As one type of non-coding RNA, long-chain non-coding RNA (lncRNA) can regulate gene expression on multiple levels, such as the epigenetic, transcription, and posttranscription levels ${ }^{5-7}$. For example, one study showed that LINC01234 activated VAV3 at the post-transcription level and repressed BTG2 at the transcription level, which subsequently led to epithelial-to-mesenchymal transition in NSCLC cells. Additionally, lncRNA SNHG20 promoted proliferation and migration by epigenetically silencing $\mathrm{P} 21^{8}$.

\section{(c) The Author(s) 2020}

(c) (i) Open Access This article is licensed under a Creative Commons Attribution 4.0 International License, which permits use, sharing, adaptation, distribution and reproduction cc) in any medium or format, as long as you give appropriate credit to the original author(s) and the source, provide a link to the Creative Commons license, and indicate if changes were made. The images or other third party material in this article are included in the article's Creative Commons license, unless indicated otherwise in a credit line to the material. If material is not included in the article's Creative Commons license and your intended use is not permitted by statutory regulation or exceeds the permitted use, you will need to obtain permission directly from the copyright holder. To view a copy of this license, visit http://creativecommons.org/licenses/by/4.0/. 


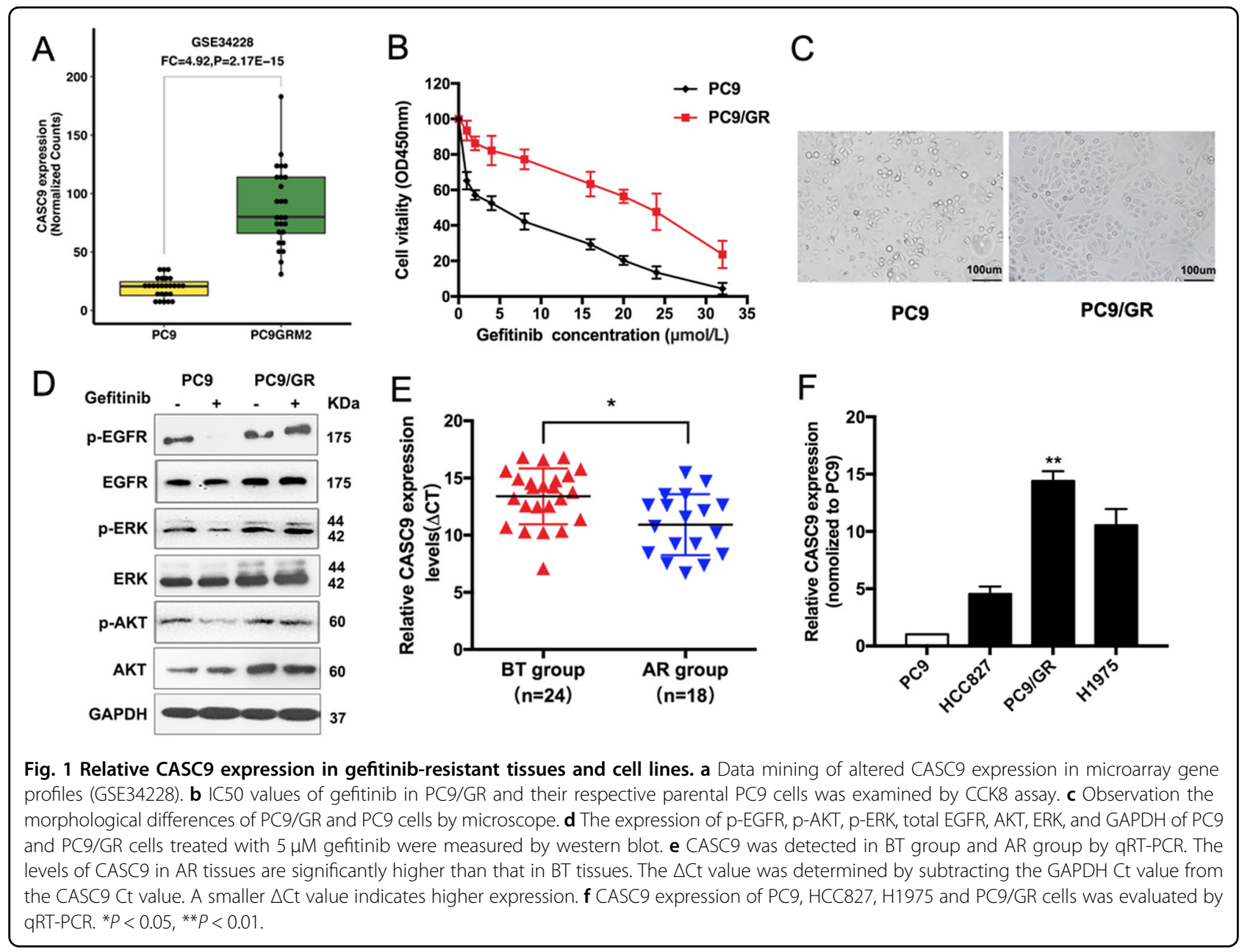

IncRNAs also form complex regulatory networks in cells ${ }^{9}$, which provides a new research direction for drug resistance in NSCLC. IncRNAs play a key role in the development and formation of tumor resistance ${ }^{10-13}$. lncRNA UCA1 promotes gefitinib resistance by sponging miR-143 in NSCLC ${ }^{14}$, and lncRNA CCAT1 acts as an miR-218 sponge to increase gefitinib resistance by targeting HOXA $1^{15}$. Our previous study revealed that LINC01116 facilitates gefitinib resistance by downregulating IFI $44^{13}$. As a result, IncRNAs have been highlighted as novel players in tumor resistance. However, only a small fraction of lncRNAs and their role in drug resistance have been elucidated, and much information remains unknown.

In the present study, we performed lncRNA profiling of GEO datasets and identified CASC9, which was overexpressed in gefitinib-resistant NSCLC cells and tissues. CASC9 promoted gefitinib resistance both in vitro and in vivo. We further uncovered the underlying mechanism and proposed that CASC9 may be a novel predictive biomarker and therapeutic target for NSCLC gefitinib resistance.

\section{Results}

CASC9 is overexpressed in gefitinib-resistant PC9/GR cells and correlated with acquired resistance to gefitinib

To identify the mechanisms that are involved in acquired resistance to EGFR-TKIs, we conducted an integrative analysis of lncRNAs/mRNA for PC9 and PC9/ GR cells of GSE34228 from the GEO datasets. CASC9 expression was higher in PC9/GR cells with acquired resistance to gefitinib (Fig. 1a). To explore the correlation between CASC9 and EGFR-TKIs, we established gefitinibresistant (GR) NSCLC cells by exposing PC9 cells to increasing concentrations of gefitinib for over 6 months. The inhibitory concentration of gefitinib yielding $50 \%$ cell viability (IC50) was calculated from linear dose-response curves using the CCK8 assay (Fig. 1b). We used high power microscopy to observe the morphology of PC9 and PC9/GR cells (Fig. 1c). Next, we evaluated the effects of gefitinib on the phosphorylation of EGFR, ERK, and PI3K/ $\mathrm{AKT}$, three major downstream pathways that activate in PC9/GR cells. Western blot results showed that after gefitinib treatment, there was no significant change in the phosphorylation of EGFR, ERK, and AKT in PC9/GR cells, 


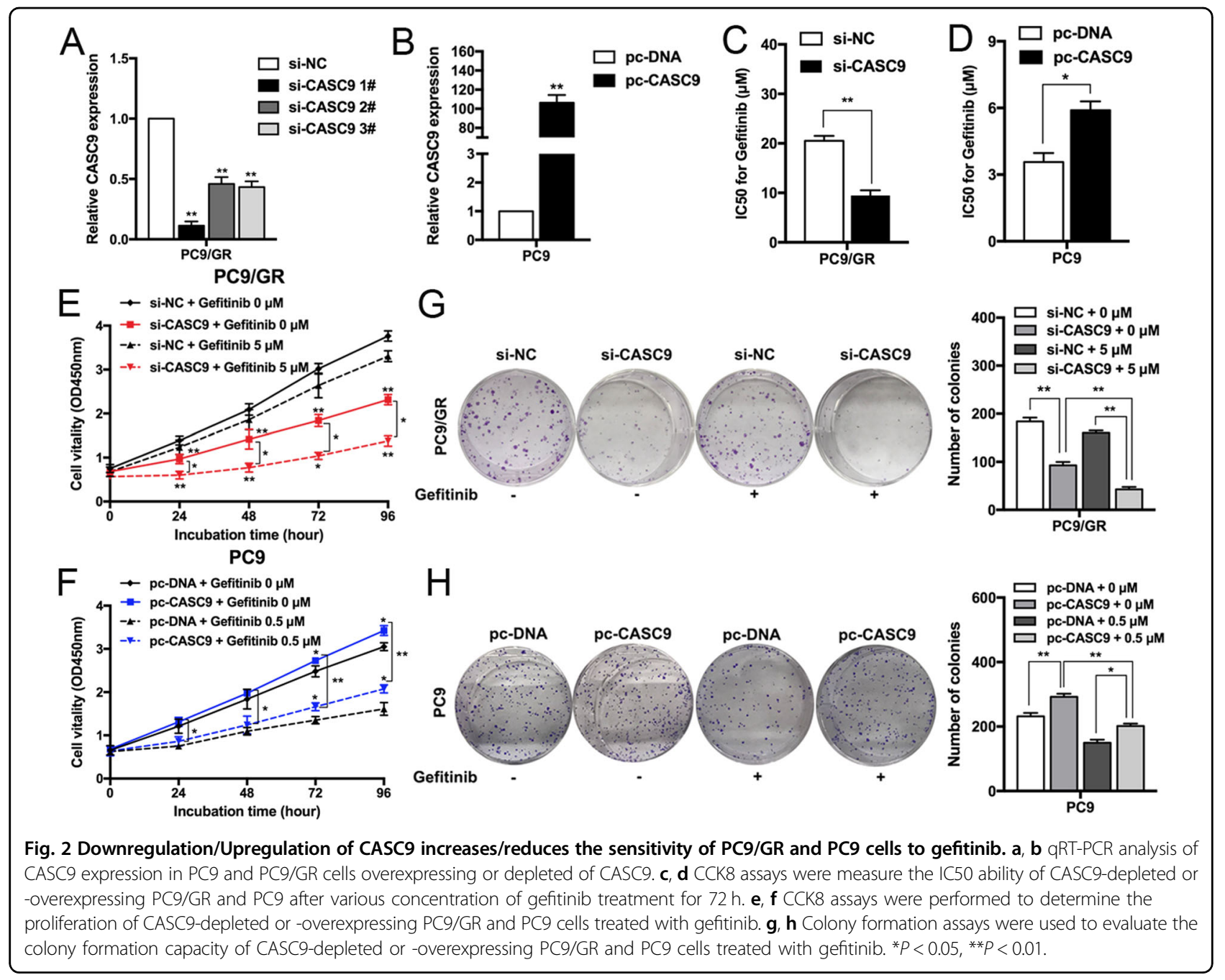

while the phosphorylation of EGFR, ERK, and AKT in PC9 cells was significantly inhibited (Fig. 1d and Supplementary Fig. S1a). These findings suggested that there is a bypass in PC9/GR cells to activate the EGFR downstream signal. Furthermore, we examined CASC9 expression in developing resistance to EGFR-TKIs in advanced NSCLC patients, which were divided into two groups (Supplementary Table S1): before EGFR-TKI treatment (defined as the BT group) and acquired resistance to EGFR-TKIs (defined as the AR group). qRT-PCR analysis showed that CASC9 expression in the AR group was significantly higher than in the BT group (Fig. 1e). In NSCLC cell lines, CASC9 expression was higher in TKI acquired resistance cells (PC9/GR and H1975) than in the TKI-sensitive cell line (PC9 and HCC827) (Fig. 1f).

\section{CASC9 resensitizes gefitinib-resistant NSCLC to gefitinib in vitro}

To explore the biological functions of CASC9 in the sensitivity of NSCLC cells, we transfected PC9/GR cells with CASC9-specific small interfering RNA (siRNA) to diminish its expression (Fig. 2a), and a CASC9 overexpression vector was transfected into parental PC9 cells to upregulate its expression (Fig. 2b). CCK8 assays showed that CASC9 downregulation significantly inhibited the IC50 value of PC9/GR cells to gefitinib (Fig. 2c), and that CASC9 overexpression induced PC9 cell resistance to gefitinib (Fig. 2d). To further confirm the role of CASC9 in gefitinib resistance, we constructed CASC9 overexpression vector with binding sites of si-CASC9 mutated. Cotransfection with CASC9 mutated vector and si-CASC9 showed higher IC50 value compared with co-transfection with empty vector and si-CASC9 (Supplementary Fig. S1b and c). Next, we determined the effects of CASC9 on cell proliferation. As shown in Fig. 2e, f, CCK8 proliferation assays showed that CASC9 downregulation significantly inhibited PC9/GR cell proliferation compared with the control cells and, conversely, CASC9 overexpression promoted PC9 cell proliferation with or without gefitinib treatment. Similar results were obtained when PC9/GR or 


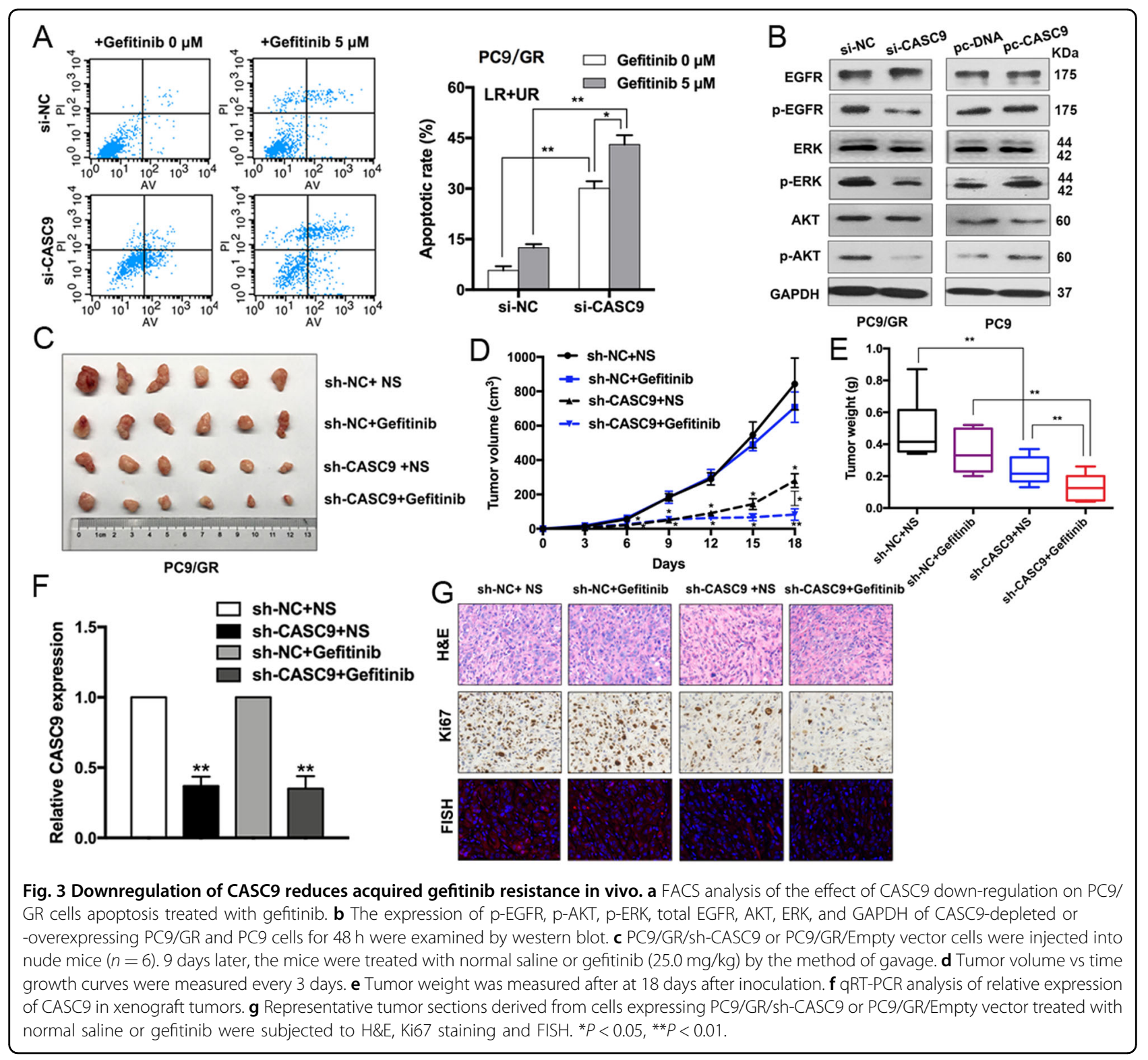

PC9 cell clonogenic survival potential was measured with colony-forming assays (Fig. 2g, h).

We also performed flow cytometry to investigate whether CASC9 is involved in regulating cell apoptosis. Compared with the control cells, CASC9 knockdown significantly increased the PC9/GR cell apoptotic rate with or without gefitinib treatment (Fig. 3a). We next examined whether CASC9 regulates ERK and AKT downstream signaling. CASC9 depletion resulted in significantly and markedly decreased phosphorylation levels of EGFR, AKT, and ERK in PC9/GR cells, whereas CASC9 overexpression dramatically increased EGFR, ERK, and AKT signaling in PC9 cells (Fig. 3b). Overall, these data confirmed that CASC9 can make drug-resistant cells sensitive to gefitinib in vitro.

\section{CASC9 knockdown suppresses gefitinib-resistant cancer development in vivo}

To further validate the role of CASC9 in PC9/GR cell resistance to gefitinib in vivo, we injected the CASC9 stable knockdown PC9/GR cells or control cells into nude mice to employ a mouse xenograft model. As shown in Fig. 3c-e, CASC9 knockdown repressed tumor growth and weight, especially in cells treated with gefitinib. qRT-PCR analysis confirmed that CASC9 expression was inhibited in the excised tumors (Fig. 3f). Compared with the control tumors, sh-CASC9-derived tumors expressed lower levels of the proliferation marker Ki-67 as assessed by IHC staining and lower levels of CASC9 by fluorescence in situ hybridization (FISH) (Fig. 3g). 


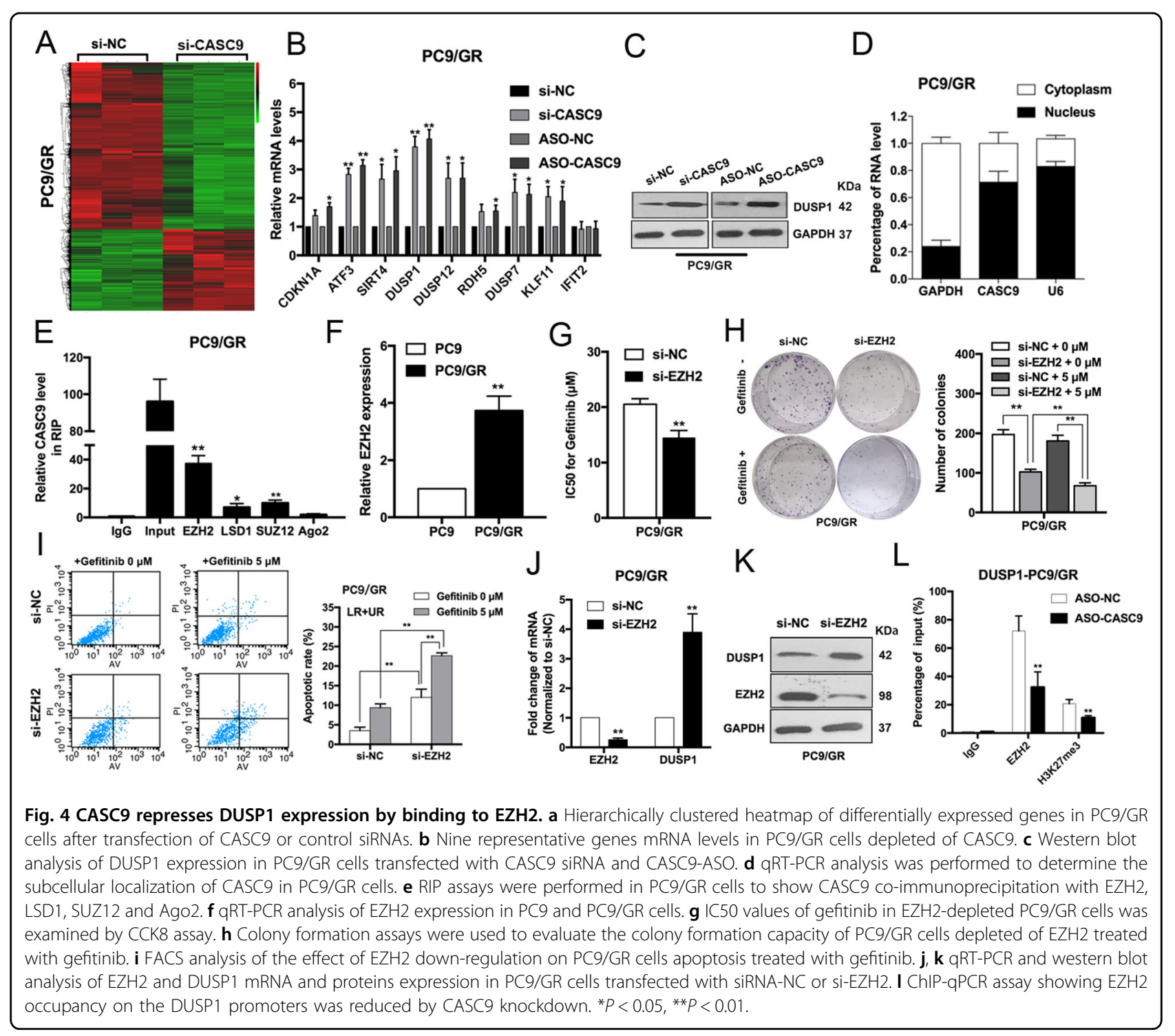

CASC9 drives gefitinib resistance via increasing EZH2 and activating the ERK pathway

The ERK pathway is of great importance in NSCLC cell proliferation, metastasis, and drug resistance ${ }^{16}$. To better understand the underlying molecular mechanism of CASC9 in acquired resistance to gefitinib, we used an RNA-sequencing analysis. To confirm the function of CASC9, we also designed LNA-ASO targeting CASC9. qRT-PCR studies confirmed that transfection with CASC9-ASO efficiently reduced CASC9 expression in PC9/GR cells (Supplementary Fig. S1d). As shown in Fig. 4a, expression of a large number of genes was upregulated in cells with CASC9 knockdown. We chose those genes involved in drug resistance to validate RNA-seq results. DUSP1 attracted our attention because of the highest-fold upregulation in CASC9 silencing PC9/GR cells (Fig. 4b). Western blot analysis subsequently confirmed that CASC9 knockdown significantly upregulated DUSP1 protein levels in PC9/GR cells (Fig. 4c). Additionally, we analyzed the distribution of CASC9 in PC9/GR cells using subcellular fractionation analyses. qRT-PCR analysis showed that CASC9 is distributed in both the cytoplasm and nucleus, but the ratio of CASC9 in the nucleus is higher (Fig. 4d), indicating that CASC9 may function as a regulator at the transcription level.

Recent studies have indicated that lncRNAs can regulate the expression of downstream targets by interacting with RNA binding proteins, such as EZH2, SUZ12, and LSD ${ }^{12,17}$. To determine whether CASC9 regulates the potential targets by binding these proteins, we performed RNA immunoprecipitation (RIP) assays and confirmed that CASC9 is able to bind with EZH2, SUZ12, and LSD1. However, CASC9's interaction with EZH2 was stronger, indicating that CASC9 interacted specifically with EZH2 
in PC9/GR cells (Fig. 4e). To further study the interaction between CASC9 and EZH2, we examined the EZH2 expression by qRT-PCR and western blot. No significant changes were observed in si-CASC9 cells compared with negative control (Supplementary Fig. S1e, f).

EZH2, a core subunit of polycomb repressive complex 2 (PRC2), plays a vital role in treatment resistance in NSCLC $^{18,19}$. To determine whether EZH2 is associated with gefitinib resistance, we examined EZH2 expression in PC9 and PC9/GR cells. The expression level of EZH2 was found to have a higher expression level in resistant cells of GSE34228 from GEO datasets (Supplementary Fig. S2a). Expression level of EZH2 was upregulated in PC9/GR cells compared with PC9 cells (Fig. 4f, Supplementary Fig. S2c, d). EZH2 knockdown restored gefitinib sensitivity, inhibited cell proliferation, and induced apoptosis in PC9/GR cells (Fig. 4g-i). In addition, we observed that the loss of EZH2 was related to DUSP1 upregulation at the mRNA and protein level (Fig. 4j, k). We then conducted chromatin immunoprecipitation assays and found that EZH2 could bind directly to the promoter regions of DUSP1 and induce H3K27 trimethylation. Furthermore, CASC9 knockdown decreased the binding of EZH2 and H3K27 trimethylation levels across the promoters of DUSP1 (Fig. 4l). These results suggest that CASC9 affects gefitinib resistance, at least partly, through the epigenetic repression of DUSP1 by interacting with EZH2 in PC9/GR cells.

\section{DUSP1 upregulation restored PC9/GR cell sensitivity to gefitinib}

To further verify the potential role of DUSP1 in PC9/GR cells, we examined its expression in PC9 and PC9/GR cells. DUSP1 was also found to be downregulated in resistant cells of GSE34228 from GEO datasets (Supplementary Fig. S2b). Expression level of DUSP1 was upregulated in PC9 cells compared with PC9/GR cells (Fig. 5a, Supplementary Fig. S2c, d). DUSP1 expression was upregulated when we used an overexpression vector (Fig. 5b), and DUSP1 overexpression reduced the IC50 of PC9/GR cells treated with gefitinib (Fig. 5c). CCK8 and colony formation assays revealed that DUSP1 overexpression inhibited PC9/GR cell proliferation with or without gefitinib treatment (Fig. 5d, e). Moreover, flow cytometry showed that the proportion of apoptotic cells increased significantly following treatment with a DUSP1 vector (Fig. 5f). We also discovered that DUSP1 overexpression markedly decreased the expression of phosphorylationERK (p-ERK), indicating that the ERK signaling pathway was inactivated, by western blot (Fig. 5g). Additionally, we detected the mRNA and protein levels of EZH2 and DUSP1 in the xenograft tumors after sh-CASC9 and gefitinib treatment. There were no significant differences of expression levels of EZH2 in those groups. DUSP1 was upregulated with knockdown of CASC9 in the presence or absence of gefitinib (Supplementary Fig. S2e-g).

\section{CASC9 contributes to acquired resistance to gefitinib and is partly dependent on regulating DUSP1}

Next, we identified whether suppressing DUSP1 expression could restore the effects of CASC9 knockdown on regaining gefitinib sensitivity in PC9/GR cells. As shown in Fig. 6a, si-CASC9 expression significantly increased DUSP1 mRNA levels, and these effects were reversed by co-expression of a DUSP1 inhibiter. The increased DUSP1 expression and decreased p-ERK protein induced by si-CASC 9 were also partly rescued by cotransfection with a DUSP1 inhibitor (Fig. 6b). Moreover, si-DUSP1 reversed the CASC9 knockdown-induced decrease in the IC50 of gefitinib in PC9/GR cells (Fig. 6c). Furthermore, DUSP1 downregulation reversed the effects of si-CASC9 on proliferation capacity in PC9/ GR cells (Fig. 6d, e). Collectively, these data indicated that the CASC9-EZH2-DUSP1 axis regulates p-ERK expression to promote gefitinib resistance in NSCLC (Fig. 6f).

\section{Discussion}

The mechanism of EGFR-TKI acquired resistance is very complex. Currently, the reported reasons for EGFR-TKI acquired resistance are EGFR 20 exon T790M mutation, MET amplification, and phenotypic transformation of tumor cells ${ }^{20-23}$. However, secondary drug resistance in tumors is caused by the regulation of a multi-factor network, and there is still a lack of clinically applicable efficacy prediction markers and effective intervention strategies based on drug resistance molecular mechanisms ${ }^{24}$. Recent studies have found that ncRNA affects gene expression and translation at transcriptional and post-transcriptional levels and plays an important role in the development of drug resistance in tumor cells. In the present study, IncRNA CASC9 was screened and identified using public lncRNA expression profiles from the GEO datasets (GSE34228). We established a gefitinib-resistant cell line named PC9/GR, which has been described and reported previously ${ }^{13}$, to investigate the molecular mechanism of IncRNA-mediated gefitinib resistance. Consistent with results shown in the GEO datasets, CASC9 was expressed at a higher level in gefitinib-resistant cells and tissues compared with gefitinib-sensitive cells and tissues. In vitro and in vivo studies uncovered CASC9's important role in gefitinib resistance. Although CASC9 was upregulated and played an oncogenic role in different types of cancers ${ }^{25-28}$, the function and mechanisms of CASC9 in NSCLC gefitinib resistance has never been investigated.

A large number of recent studies have shown that lncRNAs promote gefitinib resistance through a variety of mechanisms. For example, lncRNAs induced gefitinib resistance by functioning as a competing endogenous $\mathrm{RNA}^{29}$. By recruiting histone modification enzymes or 

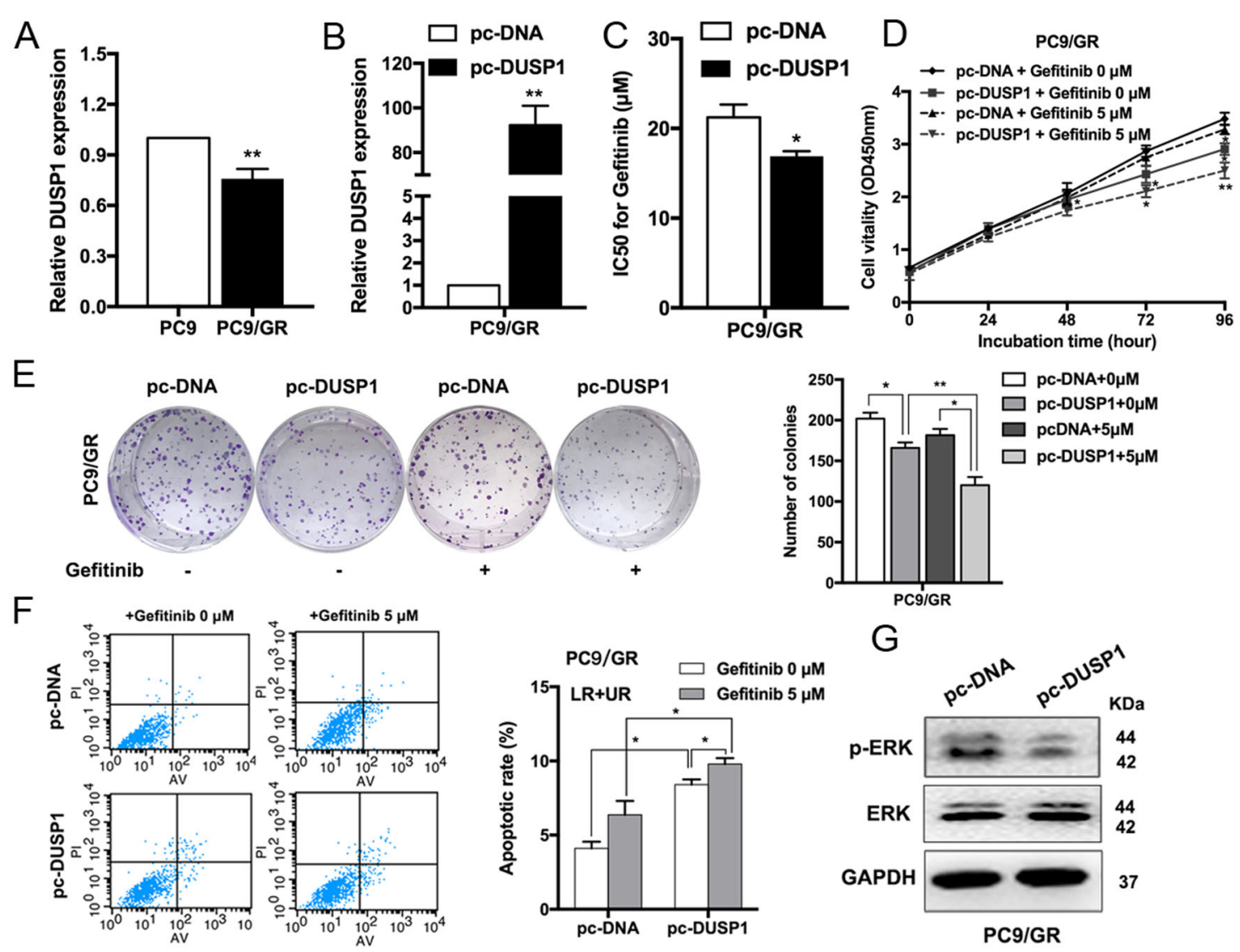

PC9/GR

Fig. 5 Upregulation of DUSP1 increases the sensitivity of PC9/GR cells to gefitinib and regulates ERK pathway. a qRT-PCR analysis of DUSP1 expression in PC9 and PC9/GR cells. $\mathbf{b}$ qRT-PCR analysis of DUSP1 mRNA levels in PC9/GR cells overexpressing DUSP1. c CCK8 assays were performed to determine the IC50 ability of DUSP1-overexpressing PC9/GR cells. $\mathbf{d}$, e CCK8 and colony formation assays were performed to determine the proliferation of DUSP1-overexpressing PC9/GR cells treated with gefitinib. $\mathbf{f}$ FACS analysis of PC9/GR cells apoptosis overexpressing DUSP1 treated with gefitinib. $\mathbf{g}$ Western blot analysis of ERK and p-ERK proteins expression in PC9/GR cells transfected with DUSP1 vector. ${ }^{*} P<0.05$, ${ }^{* *} P<0.01$.

interacting with transcription factors, lncRNAs can activate or suppress gene transcription and regulate the development of gefitinib resistance ${ }^{30-32}$. In this study, we found that CASC9 was able to bind to the histone modification enzyme, EZH2, to repress DUSP1 expression. EZH2 is the core subunit of the PRC2 complex, which inhibits target gene transcription via trimethylating of $\mathrm{H} 3 \mathrm{~K} 27^{12}$. EZH2 is involved in treatment resistance in multiple cancers. For example, EZH2 epigenetically regulates the FADD/PARP1 axis, leading to TMZ resistance in glioma ${ }^{33}$; EZH2 activates cellular survival pathways, resulting in resistance to cisplatin ${ }^{34}$; and H3K27 mediated by EZH2 induces multidrug resistance in small cell lung cancer $^{35}$. In the present study, EZH2 knockdown partially reversed gefitinib resistance, inhibited cell proliferation, and induced apoptosis in PC9/GR cells. Our results are in accordance with previous studies illustrating that EZH2 regulates gefitinib resistance in cancer cells ${ }^{36}$.

We also performed transcriptome sequencing to identify the target genes of CASC9, and we identified DUSP1 as a candidate. DUSP1 is reduced in various cancers, including liver cancer, pancreatic cancer, and lung cancer and this gene is considered a tumor suppressor that belongs to the MKP phosphatase family. DUSP1 plays an important role in regulating cell proliferation, tumorigenesis, and drug resistance ${ }^{37-39}$. Our findings indicated that EZH2 was able to bind directly to the promoter regions of DUSP1 and induce H3K27 trimethylation. CASC9 silenced DUSP1 transcription by recruiting EZH2 to the promoter region of DUSP1. DUSP1 overexpression reduced the level of $\mathrm{p}$-ERK.

ERK signaling plays a critical role in TKI resistance in NSCLC $^{40}$. The ERK-DUSP1 pathway is a phosphorylation cascade, in which ERK activation requires dual phosphorylation by a specific MAPK kinase. Each activated MAPK specifically targets many proteins, such as downstream kinases and transcription factors. Importantly, some studies have shown an association between ERK/DUSP1 pathway activation and invasive tumor phenotypes, characterized by inducing drug resistance and poor survival. In the current 


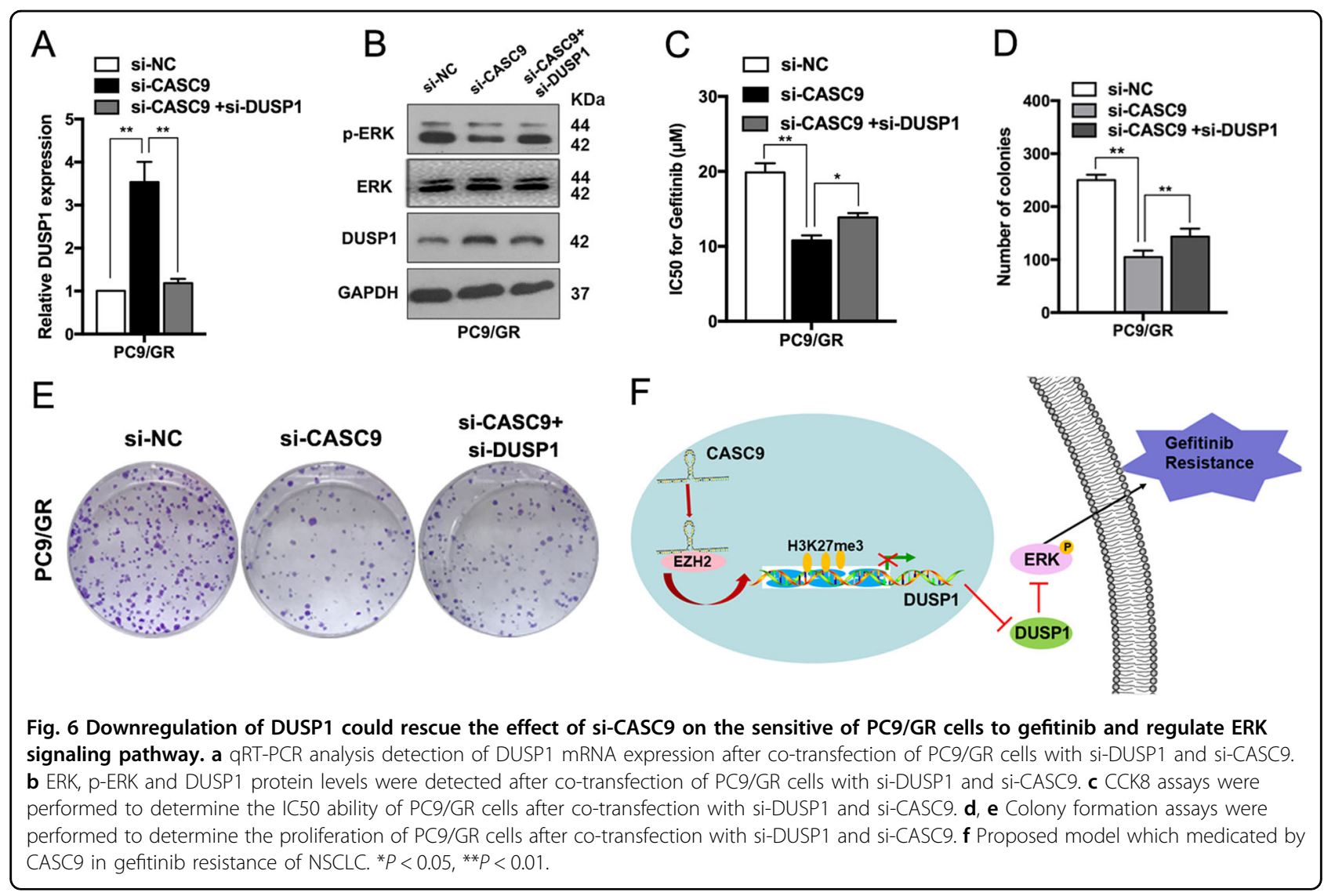

study, we also found that DUSP1 restored PC9/GR cell sensitivity to gefitinib by reducing activity in the ERK signaling pathway. Rescue experiments showed that DUSP1 knockdown reversed the effects of si-CASC9 on proliferation, gefitinib resistance, and ERK signaling activity. These results confirmed that the oncogenic function of CASC9 is partly dependent on repression of DUSP1 transcription.

In summary, the present study revealed that CASC9 promoted gefitinib resistance by recruiting the histone modification enzyme, EZH2, to repress DUSP1 expression epigenetically, which is involved in the ERK signaling pathway. These findings enhance our understanding of the CASC9/EZH2/DUSP1 axis in NSCLC gefitinib resistance.

\section{Materials and methods}

\section{Specimen and cell Lines}

A cohort of 42 NSCLC specimen tissues were collected by CT-guided percutaneous lung biopsy or fiberoptic bronchoscopy lung biopsy from advanced NSCLC patients who had either an exon 19 deletion (19DEL) or an exon 21-point mutation (L858R) in their EGFRs, and none of these patients received radiotherapy or chemotherapy before surgery. Among these 42 collected tissue samples, 24 were collected from patients before EGFR-TKIs treatment (defined as BT group), other 18 developed acquired resistance to EGFR- TKIs (defined as AR group). This study was approved by the Research Ethics Committee of The Second Affiliated Hospital of Nanjing Medical University and informed consent was obtained from all subjects. These specimens were immediately snap-frozen in liquid nitrogen and stored at $-80{ }^{\circ} \mathrm{C}$ until required. The clinical information of the patients is summarized in Supplementary Table S1.

The NSCLC cell lines PC9, HCC827 and H1975 were purchased from the Institute of Biochemistry and Cell Biology at the Chinese Academy of Sciences (Shanghai, China). The gefitinib-resistant PC9 cell line (PC9/GR) was established by stepwise escalation method: parental PC9 cell was cultured with stepwise escalation of concentration of gefitinib from $5 \mathrm{nM}$ to $5 \mu \mathrm{M}$ over 6 months. PC9 and PC9/ GR cells were cultured in DMEM, and HCC827 and H1975 were cultured in RPMI 1640 medium supplemented with $10 \%$ fetal bovine serum (FBS) and antibiotics $(100 \mathrm{U} / \mathrm{ml}$ penicillin and $100 \mathrm{mg} / \mathrm{ml}$ streptomycin) in humidified with $5 \% \mathrm{CO}_{2}$ at $37^{\circ} \mathrm{C}$. All cells were authenticated by STR profiling and tested for mycoplasma contamination.

\section{RNA extraction and qRT-PCR analyses}

Total RNA was extracted from NSCLC tissues or cells using TRIzol reagent (Invitrogen, Carlsbad, CA) following 
the manufacturer's protocol. The extracted RNA $(1.0 \mu \mathrm{g})$ was reverse transcribed to cDNA by using a Reverse Transcription Kit (Takara, Dalian, China). Real-time PCR analyses were conducted using SYBR Green (Takara, Dalian, China). Glyceraldehyde-3-phosphate dehydrogenase (GAPDH) and U6 snRNA were used as endogenous controls. The relative fold change in expression was analyzed and expressed by the $2^{-\Delta \Delta \mathrm{Ct}}$ method. The rest of primers were listed in Supplementary Table S2.

\section{Plasmid constructs and cell transfection}

CASC9 and DUSP1 cDNA were synthesized according to their coding sequences and cloned into the expression vector pcDNA3.1. Plasmids were purified using DNA Midiprep Kits (Qiagen, Valencia, CA) and transfected into PC9 and PC9/GR cells using X-treme GENE HP DNA transfection reagent (Roche, Basel, Switzerland). Three CASC9-targeting small interfering RNAs (siRNAs) (Genepharma, Shanghai, China) and three CASC9-targeting antisense oligonucleotides (ASOs) (Qiagen, Valencia, CA) were transfected into PC9/GR cells using lipofectamine 3000 (Invitrogen, Carlsbad, CA) following the manufacturer's manual. The sequences for the siRNAs and ASOs are listed in Supplementary Table S2. Cells were collected $48 \mathrm{~h}$ after transfection for quantitative real-time PCR and other individual experiments.

\section{Cell proliferation assays}

Cell proliferation was measured using the Cell Counting Kit-8 (CCK8) (APExBIO, Houston, TX). PC9 and PC9/GR cells transfected with si-CASC9, si-NC, pc-DNA or pcCASC9 were plated in 96-well microtiter plates at $1.0 \times 10^{3} /$ well, and incubated overnight. Then, the cells were treated with different concentrations of gefitinib (AstraZeneca, London, UK) for $72 \mathrm{~h}$. Subsequently, $10 \mu \mathrm{l}$ of CCK- 8 was added to each well and incubated for $2 \mathrm{~h}$, cellular viability was determined by measuring the absorbance of the converted dye at $450 \mathrm{~nm}$. For the colony-formation assay, cells were harvested at $24 \mathrm{~h}$ after transfection, then seeded into 6well plates maintaining in media containing $10 \%$ FBS and exposed to gefitinib for $48 \mathrm{~h}$. Then, the drugs were washed away and the medium was replaced every 4 days. the colonies were fixed with methanol and stained with a $0.1 \%$ crystal violet (Sigma-Aldrich) for $15 \mathrm{~min}$. Colonies were calculated. Each experiment was performed in triplicate.

\section{In vivo tumor formation assay}

For in vivo tumor formation assay, stable CASC9knockdown PC9/GR cells $\left(5 \times 10^{6}\right)$ and control cells $(5 \times$ $10^{6}$ ) were inoculated into twenty-four 5 -week-old male nude mice. 9 days after the tumor cell inoculation, mice bearing tumors were randomly assigned to 4 groups (each group with 6 mice) and gefitinib treatment was administered by oral gavage every day at a dose of $25 \mathrm{mg} / \mathrm{kg}$.
The tumor volumes and weights were measured every 3 days in mice. Tumor volume was calculated using the formula: $V=0.5 \times$ length $\times$ width $^{2}$. At 18 days post-injection, mice were euthanized, and the subcutaneous growth of each tumor was examined. All animal experiments were conducted with the approval of the Nanjing Medical University Institutional Committee for Animal Research and in conformity with national guidelines for the care and use of laboratory animals.

\section{Transcriptome sequencing}

Total RNA from PC9/GR cells with CASC9 knockdown and control cells were isolated and quantified. We used a NanoDrop 2000 (Thermo Scientific, USA) to measure the concentration of each sample and assess the quality by Agilent2200 (Agilent, USA). Ion Proton Total RNA-Seq Kit v2 was used to establish the sequencing library of each RNA sample according to the protocol provided by the manufacturer (Life Technologies, USA). Data are available in Supplementary Table S3.

\section{Flow-cytometric assay}

Cells were plated in 6-well plates, treated with gefitinib with a dose of 0 or $5 \mu \mathrm{M}$. A flow cytometry assay was performed as described previously ${ }^{10}$. The percentage of apoptotic cells was determined with Annexin V-PI/FITC staining. Cell-cycle progression was determined using propidium iodide staining.

\section{Chromatin immunoprecipitation (ChIP) assay}

ChIP assay was performed according to manufacturer's instruction (Millipore, USA) as described previously ${ }^{8}$. The antibodies for EZH2 and H3 trimethyl Lys 27 were obtained from Millipore.

\section{RNA immunoprecipitation (RIP) assay}

RIP assay was performed according to manufacturer's instruction (Millipore, USA) as described previously ${ }^{12}$. Antibodies for RIP assays of EZH2, LSD1, SUZ12 and Ago2 were purchased from Millipore.

\section{Western blot assay and antibodies}

Cell lysates were electrophoretically separated by $10 \%$ SDS-PAGE, transferred to $0.22-\mathrm{mm}$ PVDF membranes (Millipore, USA), and incubated with specific antibodies. Autoradiograms were quantified by densitometry (Tannon, China). GAPDH antibody was used as a control. Other antibodies were listed in Supplementary Table S2.

\section{Statistical analysis}

Statistical analyses were performed using SPSS 20.0 (IBM, Armonk, NY, USA) and Prism software (GraphPad, La Jolla, CA, USA). LncRNA expression levels in tissues and cell lines were compared using the Mann-Whitney $U$ 
test. For the remaining assays, differences between groups were assessed by paired, two-tailed Student's $t$-test, Wilcoxon's test, or $\chi^{2}$ test, as appropriate. $P<0.05$ was considered statistically significant.

\section{Acknowledgements}

This work was supported by grants from the National Natural Science Foundation of China (Nos. 81871871, 81802275 and 81902333), Key Research and Development plan (Social development) of science and technology department of Jiangsu Province (No. BE2019760); the Medical Innovation Team Foundation of the Jiangsu Provincial Enhancement Health Project (No. CXTDA2017021); the Science and technology development fund of Nanjing Medical University (No. NMUB2018035); "123" advantageous disciplines, core technologies and "789" excellent talent training plan of the Second Affiliated Hospital of Nanjing Medical University (No. 789ZYRC202090146); general topic of Nanjing medical science and technology development fund (No.76). We thank Jane Charbonneau, DVM, from Liwen Bianji, Edanz Group China (http:// www.liwenbianji.cn/ac), for editing the English text of a draft of this manuscript.

\section{Author contributions}

Z.X.W. and X.C. designed the study. Z.Y.C. and Q.N.C. designed the main experiments, detected the cells biological function, conducted the GRT-PCR assays, performed the statistical analysis, and wrote the manuscript. Z.X.C. and J.Y.G. participated in the design of the experiments and statistical analysis. J.L.H. established the animal model. T.Y.L. and J.Z.P. collected and organized the clinical data. J.Y.G. and W.Y.F. carried out the western blot assays. All authors read and approved the final version of the manuscript.

\section{Conflict of interest}

The authors declare that they have no conflict of interest.

\section{Publisher's note}

Springer Nature remains neutral with regard to jurisdictional claims in published maps and institutional affiliations.

Supplementary Information accompanies this paper at (https://doi.org/ 10.1038/s41419-020-03047-y).

Received: 20 May 2020 Revised: 21 September 2020 Accepted: 23 September 2020

Published online: 14 October 2020

\section{References}

1. Siegel, R. L., Miller, K. D. \& Jemal, A. Cancer statistics, 2020. CA Cancer J. Clin. 70, 7-30 (2020)

2. Yuan, M., Huang, L. L., Chen, J. H., Wu, J. \& Xu, Q. The emerging treatment landscape of targeted therapy in non-small-cell lung cancer. Signal Transduct. Target Ther. 4, 61 (2019).

3. Recondo, G., Facchinetti, F., Olaussen, K. A., Besse, B. \& Friboulet, L. Making the first move in EGFR-driven or ALK-driven NSCLC: first-generation or nextgeneration TKI? Nat. Rev. Clin. Oncol. 15, 694-708 (2018),

4. Remon, J. et al. Advanced-stage non-small cell lung cancer: advances in thoracic oncology 2018. J. Thorac. Oncol. 14, 1134-1155 (2019).

5. Huang, Z., Lei, W., Hu, H. B., Zhang, H. \& Zhu, Y. H19 promotes non-small-cell lung cancer (NSCLC) development through STAT3 signaling via sponging miR-17. J. Cell Physiol. 233, 6768-6776 (2018).

6. Qian, B. et al. LncRNA H19 serves as a ceRNA and participates in non-small cell lung cancer development by regulating microRNA-107. Eur. Rev. Med Pharm. Sci. 22, 5946-5953 (2018).

7. Fazi, B. et al. The IncRNA H19 positively affects the tumorigenic properties of glioblastoma cells and contributes to NKD1 repression through the recruitment of EZH2 on its promoter. Oncotarget 9, 15512-15525 (2018).

8. Chen, Z. et al. Long non-coding RNA SNHG20 promotes non-small cell lung cancer cell proliferation and migration by epigenetically silencing of P21 expression. Cell Death Dis. 8, e3092 (2017).
9. Schmitt, A. M. \& Chang, H. Y. Long noncoding RNAs in cancer pathways. Cancer Cell 29, 452-463 (2016).

10. Chen, $X$. et al. Long noncoding RNA LINC01234 functions as a competing endogenous RNA to regulate CBFB expression by sponging miR-204-5p in gastric cancer. Clin. Cancer Res. 24, 2002-2014 (2018).

11. Chen, Z. et al. Integrative analysis of NSCLC identifies LINC01234 as an oncogenic IncRNA that interacts with HNRNPA2B1 and regulates miR-106b biogenesis. Mol. Ther., https://doi.org/10.1016/j.ymthe.2020.03.010 (2020).

12. Chen, Z. et al. Up-regulated LINC01234 promotes non-small-cell lung cancer cell metastasis by activating VAV3 and repressing BTG2 expression. J. Hematol. Oncol. 13, 7 (2020).

13. Wang, $\mathrm{H}$. et al. Long noncoding RNA LINC01116 contributes to gefitinib resistance in non-small cell lung cancer through regulating IFI44. Mol. Ther. Nucleic Acids 19, 218-227 (2020)

14. Chen, $X$. et al. IncRNA UCA1 promotes gefitinib resistance as a ceRNA to target FOSL2 by sponging miR-143 in non-small cell lung cancer. Mol. Ther. Nucleic Acids 19, 643-653 (2020).

15. Jin, X., Liu, X., Zhang, Z. \& Guan, Y. IncRNA CCAT1 acts as a microRNA-218 sponge to increase gefitinib resistance in NSCLC by targeting HOXA1. Mol. Ther. Nucleic Acids 19, 1266-1275 (2020).

16. Maik-Rachline, G., Hacohen-Lev-Ran, A. \& Seger, R. Nuclear ERK: mechanism of translocation, substrates, and role in cancer. Int. J. Mol. Sci. 20, https://doi.org/ 10.3390/ijms20051194 (2019).

17. Zhang, $\mathrm{H}$. et al. PLK1 and HOTAIR accelerate proteasomal degradation of SUZ12 and ZNF198 during hepatitis B virus-induced liver carcinogenesis. Cancer Res. 75, 2363-2374 (2015).

18. Zhan, J. et al. HOXB13 networking with ABCG1/EZH2/Slug mediates metastasis and confers resistance to cisplatin in lung adenocarcinoma patients. Theranostics 9, 2084-2099 (2019).

19. Fillmore, C. M. et al. EZH2 inhibition sensitizes BRG1 and EGFR mutant lung tumours to Topoll inhibitors. Nature 520, 239-242 (2015)

20. Balak, M. N. et al. Novel D761Y and common secondary T790M mutations in epidermal growth factor receptor-mutant lung adenocarcinomas with acquired resistance to kinase inhibitors. Clin. Cancer Res. 12, 6494-6501 (2006).

21. Engelman, J. A. et al. MET amplification leads to gefitinib resistance in lung cancer by activating ERBB3 signaling. Science 316, 1039-1043 (2007).

22. Oser, M. G., Niederst, M. J., Sequist, L. V. \& Engelman, J. A. Transformation from non-small-cell lung cancer to small-cell lung cancer: molecular drivers and cells of origin. Lancet Oncol. 16, e165-e172 (2015).

23. $\mathrm{Yu}, \mathrm{H}$. A. et al. Analysis of tumor specimens at the time of acquired resistance to EGFR-TKI therapy in 155 patients with EGFR-mutant lung cancers. Clin. Cancer Res. 19, 2240-2247 (2013).

24. Wu, S. G. \& Shih, J. Y. Management of acquired resistance to EGFR TKI-targeted therapy in advanced non-small cell lung cancer. Mol. Cancer 17, 38 (2018).

25. Chen, Y., Li, Y. \& Gao, H. Long noncoding RNA CASC9 promotes the proliferation and metastasis of papillary thyroid cancer via sponging miR-488-3p. Cancer Med. 9, 1830-1841 (2020).

26. Ding, Y., Li, X., Zhang, Y. \& Zhang, J. Long non-coding RNA cancer susceptibility 9 (CASC9) up-regulates the expression of ERBB2 by inhibiting miR-193a$5 p$ in colorectal cancer. Cancer Manag. Res. 12, 1281-1292, https://doi.org/ 10.2147/CMAR.S234620 (2020).

27. Fang, J., Chen, W. \& Meng, X. L. LncRNA CASC9 suppressed the apoptosis of gastric cancer cells through regulating BMI1. Pathol. Oncol. Res. 26, 475-482 (2020).

28. Yang, Y., Chen, D., Liu, H. \& Yang, K. Increased expression of IncRNA CASC9 promotes tumor progression by suppressing autophagy-mediated cell apoptosis via the AKT/mTOR pathway in oral squamous cell carcinoma. Cell Death Dis. 10, 41 (2019).

29. Li, X. et al. The IncRNA RHPN1-AS1 downregulation promotes gefitinib resistance by targeting miR-299-3p/TNFSF12 pathway in NSCLC. Cell Cycle $\mathbf{1 7}$ 1772-1783 (2018)

30. Liu, X. et al. LINC00665 induces acquired resistance to gefitinib through recruiting EZH2 and activating PI3KAKT pathway in NSCLC. Mol. Ther. Nucleic Acids 16, 155-161 (2019).

31. Wu, J. et al. Reciprocal interaction of HOTAIR and SP1 together enhance the ability of Xiaoji decoction and gefitinib to inhibit EP4 expression. J. Ethnopharmacol. 237, 128-140 (2019).

32. Huang, J. et al. LncRNA SNHG15 regulates EGFR-TKI acquired resistance in lung adenocarcinoma through sponging miR-451 to upregulate MDR-1. Cell Death Dis. 11, 525 (2020). 
33. Han, B. et al. ATRX/EZH2 complex epigenetically regulates FADD/PARP1 axis, contributing to TMZ resistance in glioma. Theranostics 10, 3351-3365 (2020).

34. Sun, J. et al. miR-137 mediates the functional link between c-Myc and EZH2 that regulates cisplatin resistance in ovarian cancer. Oncogene $\mathbf{3 8}, 564-580$ (2019).

35. Fang, S. et al. H3K27me3 induces multidrug resistance in small cell lung cancer by affecting HOXA1 DNA methylation via regulation of the IncRNA HOTAIR. Ann. Transl. Med. 6, 440 (2018)

36. Katona, B. W., Liu, Y., Ma, A., Jin, J. \& Hua, X. EZH2 inhibition enhances the efficacy of an EGFR inhibitor in suppressing colon cancer cells. Cancer Biol. Ther. 15, 1677-1687 (2014).
37. Arrizabalaga, $\mathrm{O}$. et al. High expression of MKP1/DUSP1 counteracts glioma stem cell activity and mediates HDAC inhibitor response. Oncogenesis 6, 401 (2017).

38. Guo, F. et al. Deubiquitinating enzyme USP33 restrains docetaxel-induced apoptosis via stabilising the phosphatase DUSP1 in prostate cancer. Cell Death Differ., https://doi.org/10.1038/s41418-019-0473-8 (2019).

39. Pan, S. et al. Long noncoding RNA LINC01111 suppresses pancreatic cancer aggressiveness by regulating DUSP1 expression via microRNA-3924. Cell Death Dis. 10, 883 (2019).

40. Wu, D. W., Chen, C. Y., Chu, C. L. \& Lee, H. Paxillin confers resistance to tyrosine kinase inhibitors in EGFR-mutant lung cancers via modulating BIM and $\mathrm{MCl}-1$ protein stability. Oncogene 35, 621-630 (2016). 